\title{
Optimization of Heat Treatment: A Research
}

\author{
Sanjay Kumar, Rahul Garg
}

\begin{abstract}
Endurance and Improvement will be the secrets to success green fabricating is actually just really a resource of invention. Back in the last couple of years a gradual speed of evolution in heating treatment was achieved, owing to its ignorance and inadequate understandability. Just lately the market has begun eye-ing on heating treatment and surface technology whilst the absolute most vital sections ended up a lot of development and research is usually always to be performed on account of the steady desire of electricity effective, ecological friendly, optimal cost procedure. Heat treatment and surface technology would be the essential systems to improve effective usage of substances employed for vehicles, aerospace, and firearms. Once all understanding, this a critical function of heating treatment lots of complex steps such as vacuum heating healing, evolution within quenching media, laser heating curing, Electro Magnetic processing, and intensive jelling have now already been accepted that includes led in $30 \%$ boost if thickness, $2530 \%$ decrease in procedure, 30\% lowering of electricity consumption plus only ecological favorable procedure. This paper offers a brief summary of fresh tendencies of heating remedy and approach optimization having its prospective technological chances.
\end{abstract}

\section{INTRODUCTION}

Heating therapy has shifted beyond recognition because the Very First easy annealing and hardening cycles Were discovered more than 100 several decades in the past. These alterations are driven with a superior comprehension of the inherent science, rising requirement for product and caliber conditions, and social elements like ecological criteria. Additionally, industry was formed by technological improvements, like the transforming utilization of substances and advancements in production processes, that were exposed fresh chances. Figuring out in the heat therapy process that's vacuum heating therapy, intensive reduction, lead populate quench with poly alkaline glycol, Electro Magnetic processing that may have enhanced the high quality and expenditure effective lowering of cycle period plus improving ecological pleasant [1]. Vacuum heating treatment was considered a blessing to this heat cure marketplace conserving 33-50percent of cycle period when compared with atmospheric procedure. Whilst, additional advancement in social press has elevated instance thickness by approximately 30 percent together side surface complete. The initiating process unmanned processing is just one of those hard technologies by which a excellent deal of succeeding for example stride of this conversion period was realized and thus augmentation while inside the land such as return strength of metal from 1015 percent. These all

processes also have demonstrated like a measure in direction of the satisfying social responsibility, which of planning ecological favorable course of action [2].

\section{TRENDS IN CONVENTIONAL HEAT TREATING}

The theory of heat-treatment usually means that the program of temperature, time, and air to create a shift within the material's microstructure. Heating therapy approach was released at the 1970's, also as then ten years has increase and progress. Vacuum processing is now increasing over every additional tech, owing to the sizable section into this requirement for top caliber, accuracy and repeatability of character operation in more complex and demanding assistance software. Heating therapy kinds:

- Vacuum carburizing

- Vacuum nitriding

- Vacuum carbo-nitriding

- Vacuum brazing

- Vacuum sintering

\section{HIGH PRESSURE VACUUM CARBURIZING}

Carburizing causes a high-carbon transport, that leads compared with gas carburizing. Using oxygen hydrocarbons averts area oxidation in the surface. Carburizing temperatures that are Substantial greater and as much as $1000{ }^{\circ} \mathrm{C}$ contribute to expansion. There is the possibility of grain development during Allergic carburizing which may grow the capacity for regeneration from the creation of the place from the content. Nevertheless recent improvements within the metal business have demonstrated it is likely to avoid grain expansion at temperatures more than $1,050^{\circ} \mathrm{C}$ using steels using alloying elements like $\mathrm{Nb}$, Ti and $\mathrm{Al}$.

High pressure gasoline quenching is a more sterile quenching system that's lots of crucial environmental and financial advantages in comparison with fluid quenching. The quenching compounds utilized, for example nitrogen and helium, are inert and render no longer more parts residue, so prompting the demand for extra expense decision in washing machine machines or even F-I re-tracking apparatus. However, the absolute most essential benefit of petrol is really just actually an exact uniform heating transport. Even the predictability of movements throughout quenching is a lot much far more uniform and certain across the loading, consequently reducing tricky machining expenses. High pressure gas guzzling has been introduced to its heating
Revised Version Manuscript Received on 10 September, 2019.

Sanjay Kumar, Mechanical Engineering, Noida Institute of Engineering and Technology, Noida, U.P. India.(Email: research.paper@niet.co.in)

Rahul Garg, Chemical Engineering, Noida Institute of Engineering and Technology, Noida, U.P. India.(Email: research.paper@niet.co.in) 
treatment of pieces from the automotive marketplace several years past, and lots of analyses have demonstrated that stimulation might be considerably controlled instead of liquid [3].

\section{HIGH PRESSURE VACUUM CARBONITRIDING}

It's a sustainable procedure of concurrently diffusing high-pressure. This also contributes to a coating. Vacuum carbonitriding can be just really actually a substantial advancement over traditional petrol carbonitriding. This approach comprises each of the underlying advantages of advantage of computer management of top ammonia material. What's more, this technique doesn't need any extracurricular burn outs not just is that the ending item or service of top quality, but nonetheless it's less expensive compared to rival traditional petrol procedures. Carbonitriding presents lower stimulation compared carburizing. Carbonitriding can be really actually just a reversal of this method. This alteration contains ammonia in the carburizing air as a way in order to bring nitrogen into the carburized circumstance since it's currently being generated. Cases of equipment steels which are usually carbonitrided comprise SAE 10 18, 11 17, and 12L14. An average of, carbonitriding has been completed in a lowly temperature compared to carburizing, among 700 and $900^{\circ} \mathrm{C}(1300$ and $1650^{\circ} \mathrm{F}$ ), also for a much briefer period. Merge this with all the simple fact estrogen inhibits the diffusion of carbon and that which generally contributes in a more rapid instance compared to the average for carburized pieces. Even a carbonitrided instance is commonly in amongst $0.075-0.75$ mm (0.003"-0.030") profound [4]. Case thickness manage: Powerful case thickness ranges for vacuum-carburized pieces normally fluctuate from 0.010 into 0.250 in. (0.25 into 6.35 millimeter). Utmost case variant in lots might be kept within 0.005 in. (0.125 millimeters), also this is regular for aerospace and also motorsport's elements. In a single definite vacuum carbonitriding app, as from Fig. 3 that the specification known as a very trivial scenario, 0.0005 into $0.0025 \mathrm{in}$., and also the last part version attained was 0.0018 into $0.0022 \mathrm{in}$, and it is roughly 30 percent greater compared to atmospheric carburising/carbonitriding [5].

\section{MICROSTRUCTURE MODIFICATION \& RESULTS}

Magnetic-field, notably substantial magnetic industry, as tidy, strong and non-contacting energy, also has got a lot to focus in substances research field, since it could certainly behave on nuclear behavior, AFF-ect like chemical structure, microstructures and properties of substances. For ferrous metals with cycle transformations, the last bolstering results by the conversion from austenite into martensite. Even the quantity of martensite got from your conversion directly has an effect on the bolstering result. Since austenite is para-magnetic and martensite is ferromagnetic, the debut of the employed magnetic industry will surely foster the conversion and therefore boost the consequence of equally nitrogen and carbon to ferrous metals under vacuum carburizing, however, even offers the extra migration and fitting and thus exert a strong impact on

bolstering. Hence, employing a magnetic discipline to warm treatment began from the process at the late $50 \mathrm{~s}$ and the $60 \mathrm{~s}$ past century. Like fever or strain, the magnetic industry is just one of those major thermodynamic parameters which can be utilized to modify the interior energies of substances. Within the example of transformation from austenite into martensite, the Gibbs free power of martensite would be substantially diminished since it's greater magnetization in comparison to this of austenite. Whilst the magnetic field may quicken the conversion and boost the sum of martensite acquired, the linked possessions have been raised. The potential causes of its consequence of this urge magnetic area can possibly be outlined as: (inches) magnetic fields escalates the conversion driving drive and therefore enriches the conversion; (two) that the magnetostrictive influence underneath the urge magnetic field induces the interspacing in among $\mathrm{Fe}$ molecules to shift occasionally that enriches diffusion of carbon molecules and also reduces their diffusion activation electricity and so boosts the conversion; (3) the magnetic area reduces the inner power of this ferromagnetic cycle and so reduces the nucleation barrier and raises the nucleation pace. Inside this manner, the magnetic industry demonstrates a positive effect about bettering the mechanical components of their traditional supplies.

The magnetic area contains sturdy thermodynamic and kinetic impacts on transformations involving stages using different equilibrium magnetizations. Whether it's employed to a transformation by a very minimal magnetization stage into your top magnetization stage, like from case from austenite into martensite, bainite or ferrite, it enriches the conversion temperature and hastens the conversion rate and raises the sum of the item period. These impacts can possibly be implemented to enhance the heat-treatment period, enhance endurance and so boost the traditional heat therapy method procedures. Moreover, the Magnetic-field will demonstrably reevaluate the healing advancement of this ferrite matrix via lactic acid border migration from magnetic domain and ordering name walls. From the particular circumstance of non temperatures tempering, the magnetic field may transform the precipitation arrangement of changeover carbides by altering their Gibbs free energies throughout magnetization and thus enhance the influence durability of this substance [8].

\section{FUTURE OF HEAT TREATMENT}

The potential for heat-treatment is incredibly glowing and thus development inside it's at an immediate rate. In just future that's at the calendar year 2020 some radical shift in tech of heating treatment could occur leading in the creation of electricity efficient, affordable, ecological friendly procedure and substances. Back in 2020 subsequent points will likely probably be attained:

Loss in generation price by 75 percent.

Upsurge in suburban lifestyle by 10 folds.

Reduction in price by 50 percent of the furnace.

Lowering of energy usage by 80 percent. 
Reach zero stimulation and highest possible uniformity in heating cured course of action.

Advancement of hybrid gas/electric heating approach to reduce electricity price. Advancement of steels for induction which enables much speedier processing intervals. Advancement of substances appropriate for speedy heating technology [9].

\section{CONCLUSION}

Efforts in the direction of the evolution of production and design engineering of lighting, lasting and ecological friendly substances will soon probably go farther and rivalry from material generally appears to eventually become increasingly intense. Regardless of the prevailing popular heating procedures, there's potential to grow electricity and processing efficiency and enhance the solution or service quality from the progression of complex processing procedures. To meet and get over those challenges, we have to think within such a fashion, "All inventions are constructed on rejections". Innovation is just one of the items that culture looks says $\&$ at if we create this portion of just how we work $\&$ live, it is going to modify the way in which we work \& live. So finishing that nevertheless you'll find respective barriers in the course of achievements however heating treatment from 2020 is going to soon be in its own landmark.

\section{REFERENCES}

1. V. K. Manchanda, G.B.S. Narang, 2005. "Materials and Metallurgy", 6th Edition, Khanna Publishers.

2. K.W.K. Yeunga, K.M.C. Cheunga, W.W. Lua, C.Y. Chungb, "Materials Science and Engineering" A, 383 (2004) 213-218

3. Sidney H.Avner, 2010. "Introduction to Physical Metallurgy", Second Edition, Tata McGraw-Hill.

4. Htuni, G.G., M. Faccoli, and M. Roberti. 2009. "Influence of Aging on Microstructure". 198-205.

5. Herring, D.L. and V. Jenko. 2009. "Estimation of Fracture Toughness of Nitride Compound Layers on Tool Steel by Application of Vickers Indentation Method". Surface and Coating Technology. 201:182-188.

6. K. Funatani, G.E. Totten, "Present Accomplishments and Future Challenges of Quenching Technology", Proceedings of the 6th International Federation of Heat treatment and Surface Engineering Congress, IFHTSE, Kyongju, Korea, 1997, 20-27.

7. Nam W. J. \& Bae C. M. (1999), "Coarsening Behavior of Cementite Particles at a Subcritical temperature in a medium Carbon Steel". Scripta Materialia, Vol. 41, No. 3, pp. 313-318.

8. Daniel Herring, "Technology trends in vacuum heat treating ,part three: new technology and future developments" ,Midwest thermal -vac Kenosha, January 2009(online).

Available: http://www.heat-treat-doctor.com.

9. Donald Jordan, "Vacuum gas nitriding furnace produces precision nitrided parts" ,ASM Heat treating society, September 2009 [online]. Available: http://www.asminternational.com. 